\title{
AVALIAÇÃO DA TEMPERATURA DO TELHADO DE DIFERENTES MATERIAIS DE COBERTURA PARA CONSTRUÇÃO DO TELHADO DE AVIÁRIOS MÓVEIS
}

\author{
Rosimar Farias'; Maressa Fideles Pereira1; Andressa Santana Costa1; Eloinny Karina Figueira Castro'; Max Nei \\ Braz Sousa'; Natanael Costa dos Santos'; Graciene Conceição dos Santos². \\ ${ }^{1}$ Acadêmico (a) de Zootecnia, Instituto de Biodiversidade e Florestas (IBEF), Universidade Federal do Oeste \\ do Pará (UFOPA), Santarém, Pará, Brasil, roseborari@gmail.com, fidelispereira19@hotmail.com, \\ kika.andressa@gmail.com, eloinnykarina@gmail.com, maxnei.braz@hotmail.com, \\ natanaelcosta.santos@hotmail.com
}

2Docente e pesquisador (a),IBEF, UFOPA, Santarém, Pará, Brasil, gracienecsantos@yahoo.com.br

RESUMO: o presente estudo objetivou avaliar a temperatura superficial externa, interna e a diferença das temperaturas em diferentes tipos de materiais para cobertura de aviários móveis. O experimento foi realizado no período de 22 a 31 de agosto de 2016, no Campus Tapajós da UFOPA, no município de Santarém - PA. Foram construídos quatro aviários móveis. Os tratamentos referem-se aos tipos de coberturas: 1) palha; 2) Tetra Pak ${ }^{\circledR}$; 3) lona plástica dupla face; e 4) telha de amianto ou zinco. Foram feitas coletas a cada 2 horas, de 8 às 18 horas, com a utilização de termômetro infravermelho e os dados submetidos para análise estatística do pacote SISVAR pelo teste Scott-Knot a 5\% de probabilidade. Observou-se diferença estatística para temperatura superficial externa nos horários de 16 e 18 horas. Não houve diferença estatística para temperatura superficial interna dos telhados. Foi constatada diferença estatística entre os tratamentos Tetra Pak ${ }^{\circledR}$ e palha, nos horários de 10 e 16 horas, respectivamente. Conclui-se que o telhado construído a base de embalagens Tetra Pak ${ }^{\circledR}$ podem ser boas alternativas para cobertura de aviários móveis.

PALAVRAS-CHAVE: Avicultura, Bioclimatologia, Frangos de corte.

\section{EVALUATION OF THE TEMPERATURE OF THE ROOF OF DIFFERENT ROOFING MATERIALS FOR ROOF CONSTRUCTION OF MOBILE AVIARIES}

ABSTRACT: the present study aimed to evaluate the external, internal surface temperature
and the difference of these temperatures in different types of materials to cover mobile
aviaries. The experiment was carried out from August 22 to 31, 2016, at the Tapajós Campus
of UFOPA, in the city of Santarém - PA. Four mobile aviaries were built. The treatments refer
to the types of coverages: 1 ) straw; 2 ) Tetra Pak ${ }^{\circledR} ; 3$ ) double-sided plastic canvas; and 4)
asbestos or zinc tile. Data were collected every 2 hours, between 8:00 am and 06:00 p.m.,
using an infrared thermometer and the data submitted for statistical analysis of the SISVAR
package by the Scott-Knot test at 5\% probability. Statistical difference was observed for
external surface temperature in the hours of 04:00 p.m. and 06:00 p.m. There was no
statistical difference for the internal surface temperature of the roofs. A statistical difference
was observed between the Tetra Pak ${ }^{\circledR}$ and straw treatments, at the hours of 10:00 am and 
04:00 pm, respectively. Considering this, it is concluded that roofs constructed from Tetra Pak ${ }^{\circledR}$ packaging can be good alternatives for covering mobile aviaries.

KEYWORDS: Bioclimatology, Broilers, Poultry.

\section{EVALUACIÓN DE LA TEMPERATURA DEL TEJADO DE DIFERENTES MATERIALES DE COBERTURA PARA LA CONSTRUCCIÓN DEL TEJADO DE AVIONES MÓVILES}

RESUMEN: el presente estudio objetivó evaluar la temperatura superficial externa, interna y la diferencia de estas temperaturas en diferentes tipos de materiales utilizables como cobertura de aviarios móviles. El experimento fue realizado en el período del 22 al 31 de agosto de 2016, en el Campus Tapajós de la UFOPA, en la ciudad de Santarém - PA. Se construyeron cuatro aviones móviles. Los tratamientos se refieren a los tipos de cubiertas: 1) paja; 2) Tetra Pak ${ }^{\circledR}$; 3) Iona plástica doble cara; y 4) teja de amianto o cinc. Se realizaron colectas de datos cada 2 horas, entre las 8:00 am y las 06:00 pm, con la utilización de termómetro infrarrojo los datos fueron sometidos análisis estadísticos del paquete SISVAR por la prueba Scott-Knot al 5\% de probabilidad. Se observó diferencia estadística para temperatura superficial externa en los horarios de 04:00 pm y 06:00 pm. No hubo diferencia de importancia estadística para la temperatura superficial interna de los tejados. Sin embargo, se constató diferencia estadística entre los tratamientos Tetra Pak ${ }^{\circledR}$ y paja, en los horarios de 10:00 am y 04:00 pm, respectivamente. Considerando esto, se concluye que los tejados construidos a base de envases Tetra Pak ${ }^{\circledR}$ pueden ser buenas alternativas para la cobertura de los aviones móviles.

PALABRAS CLAVE: Avicultura, Bioclimatología, Pollos de corte.

No contexto atual, a avicultura de corte brasileira é um exemplo de atividade de grande importância, sendo o setor do agronegócio que mais se destaca, e a ambiência adequada é um parâmetro de extrema relevância para se obter melhores rendimentos na criação (BARBOSA, et al., 2017).
Apesar do alto potencial para produtividade, a criação de frangos ainda enfrenta desafios, mostrando-se susceptível a um grande número de variáveis, com destaque para os fatores ambientais, os quais influenciam diretamente na expressão do potencial genéticos, na eficiência de utilização de 
nutrientes e nos aspectos sanitários (LOPES et al., 2015).

Dessa forma, algumas tecnologias alternativas de produção vêm sendo desenvolvidas, aliando ao processo produtivo as exigências de conservação do meio ambiente (MATOS JÚNIOR, 2012). Dentre essas, encontram-se os aviários móveis, constituídos de bebedouros, comedouros e coberturas que, por sua vez, podem ser deslocados periodicamente na área onde estão implantados, de modo que as aves explorem locais diferenciados (FARIA FILHO et al., 2011).

Pesquisas voltadas para o conforto térmico de instalações avícolas evidenciam que uma das principais causas do estresse calórico das aves ocorre devido à radiação solar, a qual, durante o dia, coopera substancialmente no calor que adentra a construção, e assim, a principal proteção contra insolação direta visando diminuir os níveis de desconforto térmico das aves, pode ser alcançada com a utilização de cobertura adequada (MATOS JÚNIOR, 2012).

Nesse sentido, diversos índices de conforto térmico têm sido desenvolvidos e utilizados para predizer as condições ambientais sobre os animais, sendo dependentes de algumas variáveis, como a umidade relativa, a velocidade do ar, além da temperatura, que é o principal condicionante e está diretamente relacionado com o calor produzido pelas aves, o qual é absorvido por meio da radiação e trocado pelos materiais de cobertura (FONSECA, 2010; FERNANDES, 2017).

Para a criação de animais nas condições tropicais brasileiras de temperatura, a cobertura ideal das instalações deve ter de apresentar característica considerável de isolamento térmico, possuir boa capacidade para refletir a radiação solar, ser resistente às intempéries, resistente, impermeável, de fácil manuseio e baixo custo relativo (MATOS JÚNIOR, 2012). Diante da importância de se escolher o material adequado para cobertura, o presente estudo tem como objetivo avaliar a temperatura superficial externa, interna e a diferença das temperaturas em diferentes tipos de materiais para confecção de telhados de aviários móveis. 
O experimento foi realizado no período de 22 a 31 de agosto de 2016, na Universidade Federal do Oeste do Pará (UFOPA), município de Santarém, situado próximo às coordenadas geográficas 02026'35"S e 54²42'30"W, no estado do Pará.

Foram construídos quatro aviários móveis orientados em sentidos aleatórios, com madeiras para base com $2 \mathrm{~m}$ de comprimento x 1,5 m de largura, e 1,0 m de altura, com uma taxa de lotação de 3 aves $/ \mathrm{m}^{2}$, sendo utilizados quatro tratamentos.

Os tratamentos avaliados equivalem os tipos de coberturas, a saber: 1) palha; 2) papelão revestido com caixas Tetra Pak ${ }^{\circledR}$, com a face metálica voltada para o exterior; 3) lona plástica dupla face, com a cor preta para o interior, e a cor branca voltada para o exterior; e 4) telha de amianto.

Procederam-se coletas a cada 2 horas, das 8 às 18 horas, durante 10 dias consecutivos. As variáveis analisadas foram: temperatura superficial externa do telhado (TSE); temperatura superficial interna do telhado (TSI); e a diferença entre TSE e TSI (DEI).
Para obtenção dos dados foi utilizado termômetro do tipo infravermelho com distância média de 10 a 15 cm. Após as coletas, esses foram organizados em planilhas do programa Excel e analisados por meio do programa estatístico SISVAR. Os resultados foram submetidos a análise de variância e as médias comparadas pelo teste Scott-Knot a 5\% de probabilidade e apresentados na forma de tabelas para fins de interpretação com base em fundamentação teórica.

Os resultados da TSE encontram-se na tabela 1, na qual verifica-se diferença estatística nos valores dos tratamentos avaliados.

Nos horários de 08, 10, 12 e 14 horas não houve significância nas médias encontradas nos tratamentos. Às 16 e 18 horas o tratamento tetra pak ${ }^{\circledR}$ com a face laminada voltada para cima, obteve média inferior aos demais tratamentos nos respectivos horários. Esse comportamento térmico pode ser justificado pela composição do material da caixa, que é construída de 5\% de alumínio, 20\% de plástico e 75\% de papelão (TETRA PAK $\left.{ }^{\circledR}, 2011\right)$. Por ter uma face laminada, de acordo com Fernandes 
et al (2014), a caixa longa vida mostra-se eficiente em exercer função de manta térmica, com utilização em revestimentos e, mais comumente, em forros e telhados, como uma alternativa barata e ecológica.
Os resultados da TSI encontram-se na tabela 2, na qual não se verifica diferença estatística entre os tratamentos estudados.

Tabela 1. Valores médios de temperatura superficial externa $\left({ }^{\circ} \mathrm{C}\right)$ de aviários móveis com diferentes tipos de cobertura.

\begin{tabular}{|c|c|c|c|c|c|c|}
\hline \multicolumn{7}{|c|}{ Horário } \\
\hline Tratamento & 8 & 10 & 12 & 14 & 16 & 18 \\
\hline \multicolumn{7}{|c|}{ Temperatura superficial externa - TSE } \\
\hline Palha & $25,90 a$ & $29,18 a$ & $30,71 a$ & $30,98 a$ & $32,92 b$ & $29,62 b$ \\
\hline Tetra pak ${ }^{\circledR}$ & $25,21 a$ & $27,87 a$ & $29,92 a$ & $29,44 a$ & $26,71 a$ & $26,65 a$ \\
\hline Lona & $25,81 a$ & $30,16 a$ & $31,60 a$ & $35,59 a$ & $31,43 b$ & $31,04 b$ \\
\hline Telha & $24,79 a$ & $28,47 a$ & $29,69 a$ & $31,10 a$ & $31,42 b$ & $28,96 \mathrm{~b}$ \\
\hline CV (\%) & 8,08 & 10,82 & 8,94 & 6,83 & 10,54 & 11,29 \\
\hline
\end{tabular}

Médias seguidas de letras diferentes nas colunas diferem entre si pelo teste Scott-Knott $(p<0,05)$.

Tabela 2. Valores médios de temperatura superficial interna $\left({ }^{\circ} \mathrm{C}\right)$ de aviários móveis com diferentes tipos de cobertura.

\begin{tabular}{|c|c|c|c|c|c|c|}
\hline \multicolumn{7}{|c|}{ Horário } \\
\hline Tratamento & 8 & 10 & 12 & 14 & 16 & 18 \\
\hline \multicolumn{7}{|c|}{ Temperatura superficial interna - TSI } \\
\hline Palha & $25,93 a$ & $28,75 a$ & $30,13 a$ & $30,73 a$ & $31,21 a$ & $30,29 a$ \\
\hline Tetra pak ${ }^{\circledR}$ & $25,31 a$ & $29,84 a$ & $31,45 a$ & $30,59 a$ & $30,91 a$ & $28,04 a$ \\
\hline Lona & $25,71 a$ & $28,75 a$ & $32,03 a$ & $31,13 a$ & $31,20 a$ & $31,17 a$ \\
\hline Telha & $25,26 a$ & $28,66 a$ & $29,67 a$ & $30,78 a$ & $30,67 a$ & $28,59 a$ \\
\hline CV (\%) & 9,11 & 10,41 & 9,19 & 6,02 & 8,21 & 11,74 \\
\hline
\end{tabular}

Médias seguidas de letras diferentes nas colunas diferem entre si pelo teste Scott-Knott $(p<0,05)$.

Os resultados diferem do obtido por Matos Júnior (2012), em estudo realizado na região norte de Montes Claros (MG) com clima semelhante, o qual observou diferença significativa nos valores para temperatura superficial interna, principalmente, nos horários das 10 e 18h. Apesar de não diferirem estatisticamente, 
os tratamentos nos horários das 12, 14 e 16h geraram valores superiores aos horários das 8, 10 e 18h.

Nos horários das 8, 10, 12 e 16h, o tratamento telha de amianto apresentou menores valores em comparação com os outros tratamentos. De acordo com Lopes et al. (2015), telhas de amianto são consideradas adequadas para o conforto térmico, pois apresentam baixa condutividade térmica e capacidade de calor específico alto, possuindo menor difusividade térmica.

Os resultados da diferença de TSE - TSI encontram-se na tabela 3, na qual verifica-se diferença estatística significativa nos valores entre os tratamentos.

Tabela 3. Valores médios da diferença das temperaturas superficiais externa e interna das coberturas (TSext - TSint ${ }^{\circ} \mathrm{C}$ ) de aviários móveis com diferentes tipos de materiais.

\begin{tabular}{ccccccc}
\hline \multicolumn{7}{c}{ Horário } \\
\hline Tratamento & 8 & 10 & 12 & 14 & 16 & 18 \\
\hline \multicolumn{7}{c}{ Temperatura externa - Temperatura interna } \\
\hline Palha & $-0,04 a$ & $-0,43 b$ & $-0,60 a$ & $-0,23 a$ & $2,63 b$ & $-0,67 a$ \\
Tetra pak ${ }^{\circledR}$ & $-0,10 a$ & $-1,97 a$ & $0,70 a$ & $-1,39 a$ & $-1,30 a$ & $-1,34 a$ \\
Lona & $0,10 a$ & $0,50 b$ & $0,90 a$ & $0,39 a$ & $0,46 a$ & $0,07 a$ \\
Telha & $-0,11 a$ & $0,18 b$ & $-1,11 a$ & $0,41 a$ & $2,83 b$ & $0,37 a$ \\
\hline CV (\%) & $-2719,22$ & $-630,5$ & $-8015,8$ & $-1107,39$ & $-263,51$ & $-567,61$ \\
\hline
\end{tabular}

Médias seguidas de letras diferentes nas colunas diferem entre si pelo teste Scott-Knott $(p<0,05)$.

Nos horários de 8, 12, 14 e 18 horas os tratamentos palha, Tetra Pak ${ }^{\circledR}$, Iona dupla face e telha amianto não diferenciaram estatisticamente entre si. Entretanto, nos horários das 10 e 16 horas os tratamentos Tetra Pak ${ }^{\circledR}$ e palha, respectivamente, obtiveram diferenças estatísticas.

No horário de 10 horas, o tratamento Tetra Pak ${ }^{\circledR}$ obteve diferença significativa inferior, quando comparado aos demais tratamentos no mesmo horário. Essa diferença pode ser explicada pela camada formada entre o papelão que contribuiu para a absorção de calor no ambiente interno, e as caixas longa vida com a face metálica voltada para o ambiente externo. Conforme proposto por Sarmento et al. (2005), a cor clara da superfície externa da cobertura é um artifício simples e eficiente 
na redução da temperatura da superfície interna da cobertura reduzindo em até 9 ${ }^{\circ} \mathrm{C}$ a temperatura nos horários mais quentes do dia. Com relação às camadas que compõem a cobertura de uma instalação, Tinôco (2001) enfatiza que o processo de transferência de calor de uma camada para outra ocorre em menor quantidade. Para o autor, cada camada recebe menor quantidade de calor, sendo sujeita a um menor aumento de temperatura que a camada mais externa adjacente.

Dos tratamentos analisados, às 16 horas, a telha de amianto foi o material que obteve valor significativo na diferença de temperaturas em comparação com o tratamento palha, que obteve o segundo maior valor durante o mesmo horário. No entanto, os tratamentos não apresentaram diferenças estatísticas. Sampaio et al. (2011), indicam que as telhas pintadas de cor branca na face superior promovem elevada eficiência na interceptação da radiação solar. Nääs et al. (2001) em estudo desenvolvido simulando aviários, na Faculdade de
Engenharia Agrícola (FEAGRI) da

Universidade Estadual de Campinas (UNICAMP), estado de São Paulo, também propõem o uso de pintura clara, enfatizando a pintura branca nas telhas de fibrocimento pode promover uma reflexão em torno de 70 a $88 \%$ dos raios solares. Entretanto, Passini et al (2013), ao avaliarem a intervenção do ambiente na cobertura sobre os índices de conforto térmico em frangos de corte, verificaram que somente a pintura não é eficiente.

Pode-se concluir que telhados constituídos com caixas Tetra Pak ${ }^{\circledR}$ podem ser boas opções para cobertura alternativa de aviários móveis, visando diminuir a incidência solar sobre as aves.

Se tratando em alternativas para a melhoria do conforto térmico, a utilização de pinturas claras em telhas de amianto, constitui uma delas, podendo proporcionar aumento da capacidade de reflexão, retardando assim, o calor no interior da instalação, sempre levando em conta o paisagismo do local em que a instalação se encontra inserida. 


\section{REFERÊNCIAS}

BARBOSA, R. C.; DALÓLIO, F. S.; AMORIM, M. L.; SILVA, J. N. GONZAGA, D. A. Análise de viabilidade econômica de sistemas de aquecimento de instalações agropecuárias para criação de frangos de corte. Revista Engenharia na Agricultura, v. 25, n. 03, p. 212-222, 2017.

FARIA FILHO, D. E.; VELOSO, A. L. C.; MATOS JÚNIOR, J. B.; FERNANDES, $V_{\text {.; }}$ DIAS, A. N. Criação Agroecológica de Aves em Aviários Móveis. Revista Produção Animal Avicultura, v. 49, p. 70-74, 2011.

FERNANDES, J. S.; DANIELEWICZ, R. J.; SECCO, J. Isolamento térmico de residências através da reutilização de embalagens Tetra Pak. Revista Brasileira de Extensão Universitária, v. 5, p. 13-17, 2014.

FERNANDES, T. Conforto térmico em aviários de frango de corte colonial em diferentes tipologias construtivas, Cuiabá, 2017. 96 f. Dissertação (Mestrado em Ciências Ambientais) - Universidade de Cuiabá, Departamento de Ciências Ambientais, 2017.

FONSECA, P. C. F. Efeito do manejo de cobertura sobre índice de conforto térmico, variáveis fisiológicas e desempenho de bezerros leiteiros, Anápolis, 2010. 67 f. $\quad$ Dissertação (Mestrado em Engenharia de Sistemas Agroindustriais) - Universidade Estadual de Goiás, 2010.

LOPES, J. C. O.; RIBEIRO, M. N.; LIMA, V. B. $S$ de. Estresse por calor em frangos de corte. Revista Eletrônica Nutritime, v.12, p. 4478-4487, 2015.
MATOS JÚNIOR, J. B. Avaliação de diferentes materiais de cobertura para construção do telhado de aviários móveis, Montes Claros, 2012. 70 f. Dissertação (Mestrado em Ciências Agrárias) Universidade Federal de Minas Gerais, 2012.

NÃ̃̃S, I. A.; SEVEGNANI, K. B.; MARCHETO, F. G.; ESPELHO, J. C. C.; MENEGASSI, V.; SILVA, I. J. O. Avaliação térmica de telhas de composição de celulose e betumem, pintadas de branco, em modelos de aviários com escala reduzida. Revista Engenharia Agrícola, v. 21, p. 121-126, 2001.

PASSINI, R.; ARAÚJO, M. A. G.; YASUDA, V. M.; ALMEIDA, E.A. Intervenção ambiental na cobertura e ventilação artificial sobre índices de conforto para aves de corte. Revista Brasileira de Engenharia Agrícola e Ambiental, v. 17, p. 333-338, 2013.

SAMPAIO, C. A. P.; CARDOSO, C. O.; SOUZA, G. P. Temperaturas superficiais de telhas e sua relação com o ambiente térmico. Revista Engenharia Agrícola, v. 31, p. 230-236, 2011.

SARMENTO, L. G. V.; DANTAS, R. T.; FURTADO, D. A. F.; NASCIMENTO, J. W. B.; SILVA, J. H. V. Efeito da pintura externa do telhado sobre o ambiente climático e o desempenho de frangos de corte. Revista Agropecuária Técnica, v. 26, n. 2, 2005.

TINÔCO, I. F. F. Ambiência e instalações na produção de matrizes avícolas. In: SILVA, I. J. O. Ambiência na produção de aves em clima tropical. Piracicaba: FUNEP, 2001. 